\subsection{A LIFE COURSE APPROACH TO HEALTHY AGEING: THE HALCYOn PROGRAMME}

\author{
Chair: Prof Diana J Kuh, UK \\ 01-3.1 A LIFE COURSE APPROACH TO PHYSICAL CAPABILITY
}

doi:10.1136/jech.2011.142976a.25

D Kuh, ${ }^{*}$ R Cooper. MRC Unit for Lifelong Health and Ageing, London, UK

Physical capability, the capacity to undertake the physical tasks of daily living, is an important component of healthy ageing, and can be assessed by objective tests of muscle strength and physical performance and self-reports of everyday function. There is growing evidence that social and biological factors across life affect the peak levels of physical capability achieved in adult life and/or their subsequent rate of decline, and that, in turn, physical capability predicts subsequent health and length of survival. Until recently, this evidence had been limited to separate studies.

We first present results from recent HALCyon systematic reviews and meta-analyses on lifetime determinants of physical capability, including growth and socioeconomic conditions early in life, and on the impact of impaired capability on risk of subsequent health conditions and survival time.

We then present new findings from the HALCyon cohort studies and future plans aimed at filling the research gaps identified by the systematic reviews and previous research. These include (1) the influence of age, gender and lifetime body size on physical capability; (2) the contribution of markers of muscle and neurological development to adult physical capability levels; (3) the concordance and discordance between muscle mass, strength, physical performance and limitations in everyday function.

In conclusion, understanding and promoting healthy ageing requires comparative and in-depth research on physical capability using longitudinal studies, preferably with data on characteristics across the whole of life.

\section{1-3.2 COGNITIVE CAPABILITY AND THE LIFE COURSE}

doi:10.1136/jech.2011.142976a.26

\begin{abstract}
${ }^{1} \mathrm{M}$ Richards, ${ }^{*}$ I Deary. ${ }^{1}$ MRC Unit for Lifelong Health and Ageing, London, UK;
\end{abstract} 2University of Edinburgh, Edinburgh, UK

Cognitive function shows stability of individual differences across the life course, and has a substantial heritable component. Cognitive rank can change with age through environmental influence, especially from sources that are not shared with the rearing family; although the environmental influence that is, shared with siblings attenuates from early childhood to early adulthood. A range of influences on cognitive abilities are currently under investigation by the HALCyon collaboration. In early childhood, for example, associations between neighbourhood socioeconomic status and cognitive development appear to persist at least into midlife; and education is positively associated with adult cognition over and above the influence of cognitive development. HALCyon is also investigating why cognitive development itself influences the rate of cognitive ageing in some circumstances but not others. Cognition continues to be modifiable in adulthood and later life. New HALCyon work so far suggests that certain polymorphisms that regulate genetic repair are not associated with cognitive capability in midlife and beyond, but work is also in progress on endocrine and other biomarkers. There are associations between cognitive functions in older adulthood and health-related behaviours, particularly physical exercise, smoking and dietary choice, but there is also evidence for reverse causation. True causal effects have implications for risk of dementia in later life. While returns to intervention for cognitive and emotional problems are probably greatest when the intervention occurs early, intervention to minimise cognitive decline should also be encouraged at the behavioural level throughout adulthood.

\section{1-3.3 A LIFE COURSE APPROACH TO PSYCHOLOGICAL AND SOCIAL WELL-BEING}

doi:10.1136/jech.2011.142976a.27

C Gale.* MRC Lifecourse Epidemiology Unit, Southampton, UK

The maintenance of psychological and social well-being in the face of the changes and challenges of later life is a fundamental part of healthy ageing. Understanding what influences well-being in older people could show us ways in which individuals can be helped to flourish in later life, and may have further benefits: people who are happier or more actively engaged in social activities and relationships tend to live longer and may experience a slower rate of physical and cognitive decline How older people feel and how they function socially may be influenced by factors from different stages of life. Here, we report the main findings to date from the HALCyon collaborative programme on life course influences on psychological and social well-being. We began by examining the relative importance of influences from early and later life on anxiety and depression in five cohorts. Greater neuroticism, poorer cognitive or physical function, greater disability, and poorer health were associated in cross-sectional analyses with an increased overall likelihood of anxiety or depression. Associations between lower social class, either in childhood or currently, and increased risk of anxiety or depression were no longer statistically significant after multivariable adjustment. We found no association between birthweight and anxiety or depression. We will report the results of recent analyses in which we explored the longitudinal relations between cognition and depression, and used newly collected data on well-being in six cohorts to examine the importance of common life course influences on how people feel and how they function socially.

\section{$01-3.4$ \\ AN EPIDEMIOLOGICAL PERSPECTIVE ON BIOLOGICAL MODELS OF AGEING}

doi:10.1136/jech.2011.142976a.28

${ }^{1}$ Y Ben-Shlomo, ${ }^{2} \mathrm{~T}$ von Zglinicki, ${ }^{1} \mathrm{I}$ Day. ${ }^{1}$ University of Bristol, Bristol, UK; ${ }^{2}$ Newcastle University, Newcastle upon Tyne, UK

There a wide variety of biological theories of ageing from macrolevel concepts such as evolutionary trade-offs and reliability theory to system-based theories of neuroendocrine and immune function and molecular and cellular theories such as the role of a biological clock. Animal studies can elegantly demonstrate the role of specific pathways such as insulin signalling but their relevance to human ageing remains controversial. Fundamentally, the loss of homeostatic control across a wide range of systems may suggest that no single pathway is necessary or sufficient for ageing. The new findings from GWAS studies of chronic diseases may also highlight commonality of pathways across phenotypes.

Within the context of the Halcyon programme we will present preliminary results around our work on telomeres, the hypothalamic pituitary axis and genetic variants on potential ageing traits. We will discuss future outputs as well the challenges of exploring these ideas within an epidemiological context.

In conclusion, while new biological understanding may enhance our ability to develop new targeted interventions, it is unlikely given the multi-faceted nature of ageing that any "magic bullet" will exist. Biological markers may be useful as additional predictors of frailty and as surrogate intermediary outcomes. Public health interventions are likely to be most effective if they focus across the life course in both maximising biological and psychosocial reserve as well as reducing adverse exposures that accelerate age-related decline in function. 